\title{
Neurobiology of Nicotine Addiction: Implications for Smoking Cessation Treatment
}

\author{
Neal L. Benowitz, MD
}

Division of Clinical Pharmacology and Experimental Therapeutics, Medical Service, San Francisco General Hospital Medical Center, San Francisco, California, USA; and Departments of Medicine, Psychiatry, and Biopharmaceutical Sciences, University of CaliforniaSan Francisco, San Francisco, California, USA

\begin{abstract}
Nicotine sustains addictive tobacco use, which in turn causes much premature disability and death. The essence of drug addiction is loss of control of drug use. Molecular biology studies suggest that the $\alpha_{4} \beta_{2}$ nicotinic acetylcholine receptor subtype is the main receptor mediating nicotine dependence. Nicotine acts on these brain nicotinic cholinergic receptors to facilitate neurotransmitter release (dopamine and others), producing pleasure, stimulation, and mood modulation. Neuroadaptation develops with repeated exposure to nicotine, resulting in tolerance to many of the effects of nicotine. When a smoker stops smoking, a nicotine withdrawal syndrome ensues, characterized by irritability, anxiety, increased eating, dysphoria, and hedonic dysregulation, among other symptoms. Smoking is also reinforced by conditioning, such that specific stimuli that are psychologically associated with smoking become cues for an urge to smoke. These include the taste and smell of tobacco, as well as particular moods, situations, and environmental cues. Pharmacotherapies to aid smoking cessation should ideally reduce nicotine withdrawal symptoms and block the reinforcing effects of nicotine obtained from smoking without causing excessive adverse effects. Further, given the important role of sensory effects of smoking and psychoactive effects of nicotine, counseling and behavioral therapies are important adjuncts to and substantially augment the benefits of pharmacotherapy. (C) 2008 Elsevier Inc. All rights reserved.
\end{abstract}

KEYWORDS: Addiction; Nicotine; $\alpha_{4} \beta_{2}$ Nicotinic Acetylcholine Receptors; Pharmacotherapy; Smoking

Use of nicotine sustains tobacco addiction, which in turn causes devastating health problems including heart disease, lung disease, and cancer. Smoking harms almost every organ of the body. ${ }^{1}$ Quitting smoking at any age leads to significant reductions in the risks associated with smoking, ${ }^{1}$ and the vast majority of smokers in the United States indicate an interest in quitting. ${ }^{2}$ Despite these facts, however, approximately $80 \%$ of smokers who attempt to quit on their own relapse within the first month of abstinence and only

Statement of conflict of interest: Please see Author Disclosures section at the end of this article.

Requests for reprints should be addressed to Neal L. Benowitz, MD, Division of Clinical Pharmacology and Experimental Therapeutics, University of California-San Francisco, Box 1220, San Francisco, California 94143-1220.

E-mail address: nbenowitz@medsfgh.ucsf.edu. about 3\% remain abstinent at 6 months. ${ }^{3}$ This illustrates the powerful force of tobacco addiction and the chronic nature of the disorder. Tobacco addiction is best considered a chronic disease, with most smokers requiring repeated interventions over time before achieving permanent abstinence.

Although most of the toxicity of smoking is related to other components of the cigarette, it is the pharmacologic effects of nicotine that produce the addiction to tobacco. An understanding of how nicotine produces addiction and influences smoking behavior provides a necessary basis for optimal smoking cessation intervention. ${ }^{4}$ This article reviews the neurobiology of nicotine addiction and withdrawal, as well as the implications for nicotine addiction therapy. Components of an earlier review on nicotine addiction are also updated in this article. ${ }^{5}$ 
Table 1 Surgeon General's criteria for drug dependence

- Primary criteria

- Highly controlled or compulsive use

- Psychoactive effects

-Drug-reinforced behavior

- Additional criteria

-Addictive behavior often involves:

Stereotypic patterns of use

Use despite harmful effects

Relapse following abstinence

$\bigcirc$ Recurrent drug cravings

-Dependence-producing drugs often produce:

O Tolerance

O Physical dependence

Pleasant (euphoriant) effects

Adapted from The Health Consequences of Smoking: Nicotine Addiction. ${ }^{6}$

\section{CHARACTERISTICS OF ADDICTION}

Distinct definitions or criteria for drug dependence or addiction have been put forth by different health organizations or authorities. In the present article, the terms "dependence" and "addiction" are used interchangeably. These terms are considered equivalent because they describe similar neurochemical and psychological processes that sustain drug use. ${ }^{6}$ Furthermore, both terms indicate loss of control over drug-taking behavior-the principal characteristic of drug addiction. Thus, once a person is addicted to a drug, it is difficult to stop using it even when there are compelling reasons to do so. Drug dependence has been defined by the World Health Organization (WHO) as "a behavioral pattern in which the use of a given psychoactive drug is given a sharply higher priority over other behaviors which once had a significantly higher value." ${ }^{\prime 7}$ In other words, the drug has come to control behavior to an extent that is considered detrimental to the individual or society.

Criteria for drug dependence were developed in the 1988 Surgeon General's report, The Health Consequences of Smoking: Nicotine Addiction (Table 1). ${ }^{6}$ In addition to the highly controlled or compulsive use of a drug, the Surgeon General's criteria require that the drug produce psychoactive effects, and there must be evidence that drug-taking behavior is reinforced by the effects of the drug. Nicotine is indeed associated with many well-known pleasurable psychoactive effects, such as arousal, relaxation, and improved mood. Also, nicotine has been shown to act as a positive reinforcer of smoking; for example, people are known to smoke only tobacco that contains nicotine, and a regular smoker modifies his or her smoking behavior to maintain a particular level of nicotine in the body. ${ }^{8,9}$

A number of additional criteria commonly associated with drug addiction are listed in the Surgeon General's report (Table 1). ${ }^{6}$ Although behaviors meeting these additional criteria are exhibited by most cigarette smokers, they are not universal in all smokers. For instance, some people, when faced with concerns about harmful effects or social pressures related to smoking, are able to quit and do not relapse. Additionally, some smokers who have quit report the absence of withdrawal symptoms. ${ }^{10}$

Another set of criteria, developed for diagnostic purposes, has been presented by the American Psychiatric Association in the Diagnostic and Statistical Manual of Mental Disorders-Fourth Edition (DSM-IV) (Table 2) ${ }^{11}$ The DSM-IV criteria have been developed for drug dependence in general, rather than specifically for nicotine dependence. However, in large part, they apply to individuals addicted to nicotine. Lastly, the Fagerström Test of Nicotine Dependence is the most widely used tool for assessing the level of tolerance to nicotine and, more globally, for assessing the severity of dependence (Table 3). ${ }^{12}$ Subjects are assigned a score based on their answers to 6 questions; the higher the score, the more dependent the smoker.

\section{EVIDENCE THAT NICOTINE IS ADDICTING}

There are many lines of evidence for tobacco addiction and the role of nicotine in sustaining tobacco addiction. The first and most basic line of evidence for tobacco addiction is that most smokers state they would like to quit smoking but are unable to do so easily. Studies indicate that, on average, smokers require 4 or more quit attempts before they are successful in achieving abstinence. ${ }^{13}$ Further, in approximately $97 \%$ of smokers, unaided quit attempts are unsuccessful for achieving long-term abstinence. ${ }^{3}$

Data for patients with medical diseases are even more compelling. Although the risk of smoking after myocardial infarction should be a powerful motivation to quit, $<50 \%$ of people quit smoking after a heart attack despite these known risks. ${ }^{14}$ Similar data are noted in patients with lung ${ }^{15}$ and laryngeal cancer. ${ }^{16}$

The role of nicotine as the pharmacologic agent that maintains tobacco addiction is well supported. It has been demonstrated that the subjective and physiologic effects of intravenous nicotine and cigarette smoking are similar. ${ }^{17}$ Nicotine also meets all of the Surgeon General's primary criteria for drug addiction (Table 1), which state that the drug must (1) promote compulsive use, (2) have psychoactive effects, and (3) reinforce its own use. ${ }^{6}$ With respect to the first point, only tobacco that contains nicotine has been consumed habitually by people over long periods of time. Neither tobacco from which nicotine has been removed nor ultra-low nicotine-yield cigarettes have received general public acceptance. In contrast, chewing tobacco and snuff, which deliver substantial amounts of nicotine, are widely used. Toward the second criterion, nicotine is known to be psychoactive and has a number of positive effects on mood and cognitive function, which are discussed in greater detail below. With respect to the last point, nicotine functions as a positive reinforcer; that is, animals and humans will administer intravenous nicotine to themselves. ${ }^{18,19}$ The reinforcing effects and the self-administration of nicotine are blocked by pretreatment with the nicotinic receptor antagonist mecamylamine. ${ }^{18}$ Furthermore, to regulate body levels of nicotine, smokers tend to adjust their smoking behavior 
Table 2 Criteria for drug dependence*

A maladaptive pattern of substance use, leading to clinically significant impairment or distress, as manifested by $\geq 3$ of the following criteria occurring at any time in the same 12-month period:

1. Tolerance, as defined by either of the following:

a. Need for markedly increased amounts of the substance to achieve intoxication or desired effect

b. Markedly diminished effect with continued use of the same amount of the substance

2. Withdrawal, as manifested by either of the following:

a. The characteristic withdrawal syndrome for the substance

b. The same (or closely related) substance is taken to relieve or avoid withdrawal symptoms

3. The substance is often taken in larger amounts or over a longer period than was intended

4. There is a persistent desire or unsuccessful efforts to cut down or control substance use

5. A great deal of time is spent in activities necessary to obtain the substance, use the substance, or recover from its effects

6. Important social, occupational, or recreational activities are given up or reduced because of substance use

7. The substance use is continued despite knowledge of having had a persistent or recurrent physical or psychological problem that is likely to have been caused or exacerbated by the substance

*Criteria from the Diagnostic and Statistical Manual of Mental Disorders-Fourth Edition (DSM-IV). ${ }^{11}$

Table 3 Fagerström test* for nicotine dependence

\begin{tabular}{lll}
\hline Questions & Answers & Points \\
\hline 1. How soon after you wake up do you & Within 5 min & 3 \\
smoke your first cigarette? & $6-30$ min & 2 \\
& $31-60$ min & 1 \\
2. Do you find it difficult to refrain from & After 60 min & 0 \\
smoking in places where it is & Yes & 1 \\
forbidden, e.g., in church, at the & No & 0 \\
library, in a cinema, etc.? & & \\
3. Which cigarette would you hate most & The first one in \\
to give up? & the morning & 1 \\
& Any other & 0 \\
4. How many cigarettes/day do you & $\leq 10$ & 0 \\
smoke? & $11-20$ & 1 \\
& $21-30$ & 2 \\
5. Do you smoke more frequently during & $\geq 31$ & 3 \\
the first hours after awakening than & Yes & 1 \\
during the rest of the day? & No & 0 \\
6. Do you smoke if you are so ill that & & Yes \\
you are in bed most of the day? & No & 1 \\
* The test has a scoring range of 0 to 10 points. & & 0 \\
Adapted from Br J Addict. ${ }^{12}$ & & \\
\end{tabular}

to compensate for changes in the availability of nicotine or in the rate of elimination of nicotine from the body. ${ }^{8,9}$ Finally, breaking one's dependence on tobacco is facilitated by replacing the nicotine derived from tobacco with nicotine administered in the form of nicotine replacement medications. $^{20}$

\section{NEUROBIOLOGY OF NICOTINE ADDICTION}

\section{Nicotinic Acetylcholine Receptors}

When a person inhales smoke from a cigarette, nicotine is distilled from the tobacco and carried in the smoke particles into the lungs, where it is absorbed rapidly into the pulmonary venous circulation. It then enters the arterial circulation and moves quickly to the brain. Nicotine diffuses readily into brain tissue, where it binds to nicotinic acetylcholine receptors (nAChRs), which are ligand-gated ion channels. When a cholinergic agonist binds to the outside of the channel, the channel opens, allowing the entry of cations, including sodium and calcium. ${ }^{21}$ These cations further activate voltage-dependent calcium channels, allowing further calcium entry.

The nAChR complex is composed of 5 subunits and is found in both the peripheral and central nervous systems. ${ }^{22}$ In the mammalian brain, there are as many as $9 \alpha$-subunits ( $\alpha_{2}$ to $\alpha_{10}$ ) and $3 \beta$-subunits $\left(\beta_{2}\right.$ to $\beta_{4}$ ). The most abundant receptor subtypes in the brains of humans are $\alpha_{4} \beta_{2}, \alpha_{3} \beta_{4}$, and $\alpha_{7}$ (homomeric). The $\alpha_{4} \beta_{2}$ receptor subtype is predom- 


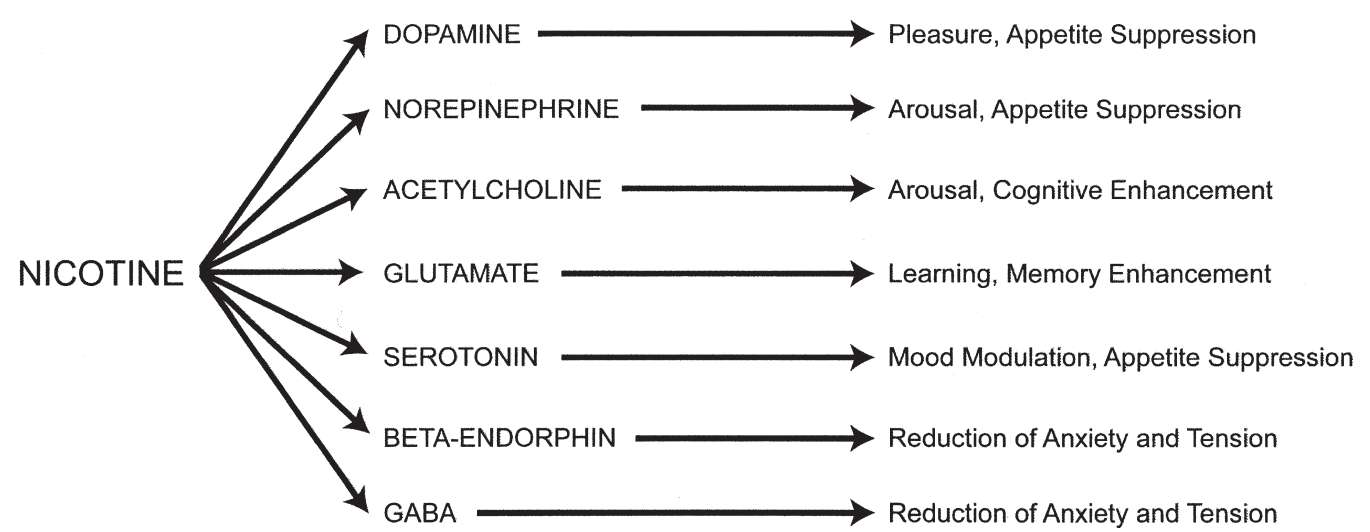

Figure 1 Nicotine receptor activation promotes the release of neurotransmitters, which may then mediate various effects of nicotine use. GABA $=\gamma$-aminobutyric acid. (Adapted with permission from Prim Care. ${ }^{5}$ )

inant in the human brain and is believed to be the main receptor mediating nicotine dependence. In mice, knocking out the $\beta_{2}$ subunit gene eliminates the behavioral effects of nicotine, including self-administration. ${ }^{23}$ Reinserting the $\beta_{2}$ subunit gene into the ventral tegmental area of a $\beta_{2}$ knockout mouse restores behavioral responses to nicotine. ${ }^{24}$ The $\alpha_{4}$ subunit appears to be an important determinant of sensitivity to nicotine. In mice, a single nucleotide point mutation in the pore-forming region results in a receptor that is hypersensitive to the effects of nicotine. ${ }^{25}$ This mutation made mice much more sensitive to nicotine-induced reward behaviors, as well as to effects on tolerance and sensitization. The $\alpha_{3} \beta_{4}$ and possibly the $\alpha_{7}$ homomeric receptor subtypes are believed to mediate the cardiovascular effects of nicotine. ${ }^{26}$ The $\alpha_{7}$ subtype is also thought to be involved in rapid synaptic transmission and may play a role in learning $^{27}$ and sensory gating. ${ }^{28}$

\section{Biology of Nicotine Reinforcement}

Brain imaging studies demonstrate that nicotine acutely increases activity in the prefrontal cortex, thalamus, and visual system, consistent with activation of corticobasal ganglia-thalamic brain circuits. ${ }^{29}$ Stimulation of central nAChRs by nicotine results in the release of a variety of neurotransmitters in the brain, most importantly dopamine. Nicotine causes the release of dopamine in the mesolimbic area, the corpus striatum, and the frontal cortex. Of particular importance are the dopaminergic neurons in the ventral tegmental area of the midbrain and the release of dopamine in the shell of the nucleus accumbens, as this pathway appears to be critical in drug-induced reward. ${ }^{22,30}$ Other neurotransmitters, including norepinephrine, acetylcholine, serotonin, $\gamma$-aminobutyric acid (GABA), glutamate, and endorphins, are released as well, mediating various behaviors of nicotine (Figure 1). ${ }^{5}$

It is believed that most of the release of neurotransmitters occurs via modulation by presynaptic nAChRs, although direct release of neurotransmitters also occurs. ${ }^{31}$ Dopamine release is facilitated by nicotine-mediated augmentation of glutamate release and, with long-term treatment, by inhibi- tion of GABA release. In addition to direct and indirect stimulation of neurotransmitter release, chronic cigarette smoking (but not acute nicotine administration) reduces brain monoamine oxidase A and B activity, which would be expected to increase monoaminergic neurotransmitter levels such as dopamine and norepinephrine in synapses, thus augmenting the effects of nicotine and contributing to addiction. $^{29}$

Dopamine release signals a pleasurable experience and is critical to the reinforcing effects of nicotine and other drugs of abuse..$^{30}$ Chemically or anatomically lesioning dopamine neurons in the brain prevents nicotine self-administration in rats. ${ }^{32}$ When intracranial self-stimulation is used as a model for brain reward in rats, nicotine acutely lowers the threshold for self-stimulation. ${ }^{33}$ Thus, through its effects on dopamine release, acute nicotine administration increases brain reward function. Likewise, nicotine withdrawal is associated with significant increases in intracranial selfstimulation reward threshold, consistent with deficient dopamine release and reduced reward. ${ }^{34}$ This is similar to the effects of withdrawal from other drugs of dependence. The decrease in brain reward function experienced during nicotine withdrawal is an essential component of nicotine addiction and a key barrier to abstinence.

With repeated exposure to nicotine, there is neuroadaptation to some, but not all, of the effects of nicotine. ${ }^{35}$ Concurrent with this neuroadaptation is an increase of nAChRs in the brain. This increase is believed to represent upregulation in response to nicotine-mediated desensitization of receptors. This desensitization may play a role in nicotine tolerance and dependence. It has been suggested that craving and withdrawal symptoms begin in chronic smokers when previously desensitized $\alpha_{4} \beta_{2}$ nAChRs become unoccupied and recover to a responsive state during periods of abstinence, such as during nighttime sleep. ${ }^{36}$ Thus, nicotine binding and desensitization of these receptors during smoking may alleviate craving and withdrawal. This is supported by clinical evidence that cigarette smoking in amounts used by typical daily smokers maintains near-complete saturation-and, thus, desensitization-of 
brain nAChRs. ${ }^{37}$ Consequently, smokers may maintain $\alpha_{4} \beta_{2}$ nAChRs in a desensitized state to avoid withdrawal. Another theory is that conditioned smoking cues maintain smoking behavior during periods of saturation and desensitization of brain nAChRs. ${ }^{38,39}$ In actuality, these 2 theories may be complementary: smokers may continue to smoke throughout the day to maintain plasma nicotine levels that prevent the occurrence of withdrawal symptoms and may also continue to derive some rewarding effects from the conditioned reinforcers associated with smoking, such as the taste and feel of the smoke. ${ }^{38}$ Conditioning as a component of addiction is discussed in more detail below.

\section{Psychoactive Effects of Nicotine and Nicotine Withdrawal}

In humans, nicotine from tobacco induces stimulation and pleasure, and reduces stress and anxiety. Smokers come to use nicotine to modulate their level of arousal and for mood control in daily life. Smoking may improve concentration, reaction time, and performance of certain tasks. When a person stops smoking, nicotine withdrawal symptoms emerge. These include irritability, depressed mood, restlessness, anxiety, problems getting along with friends and family, difficulty concentrating, increased hunger and eating, insomnia, and craving for tobacco. ${ }^{11}$ Nicotine withdrawal in untreated smokers produces mood disturbances comparable in intensity to those seen in psychiatric outpatients. ${ }^{40} \mathrm{An}$ other withdrawal symptom is hedonic dysregulation-that is, the feeling that there is little pleasure in life, and activities that were once rewarding are no longer enjoyable. This symptom is seen with withdrawal from nicotine and from other drugs of abuse. ${ }^{41}$ It is hypothesized that a relative deficiency in dopamine release following long-standing nicotine exposure accounts for many of the mood disorders and the anhedonia, as well as the tobacco craving, that may persist in smokers for a long time after they have quit.

Thus, the pharmacologic bases of nicotine addiction can be seen as a combination of positive reinforcements, such as enhancement of mood or functioning, as well as avoidance of the negative consequences of prior drug use- that is, the relief of withdrawal symptoms - in situations when nicotine is not available. In addition to these direct pharmacologic mechanisms, there is an important role for conditioning in the development of tobacco addiction.

\section{Conditioning Behavior in Nicotine Addiction}

All drug-taking behavior is learned, a result of conditioning. Drug-taking behavior is made more probable, or reinforced, by the consequences of the pharmacologic actions of the drug, as discussed for nicotine above. At the same time, the user begins to associate specific moods, situations, or environmental factors with the rewarding effects of the drug. Respiratory tract sensory cues associated with tobacco smoking represent a type of conditioned reinforcer that have been shown to play an important role in the regulation of smoke intake and the craving to smoke, as well as the rewarding effects of smoking. ${ }^{42,43}$

The association between such cues and anticipated drug effects and the resulting urge to use the drug is a type of conditioning. Animal studies find that nicotine exposure increases the behavioral control of conditioned stimuli, which may contribute to the compulsivity of smoking behavior. ${ }^{44}$ Furthermore, experimental studies in nicotinedependent rats show that nicotine withdrawal-associated conditioned stimuli potentiate the magnitude of nicotine withdrawal, including an elevation of brain reward threshold. Thus, cues associated with nicotine withdrawal have the ability to decrease brain reward function. ${ }^{45}$

Cigarette smoking is maintained, in part, by such conditioning. People habitually smoke cigarettes in specific situations, such as after a meal, with a cup of coffee or an alcoholic drink, or with friends who smoke. The association between smoking and these other events repeated many times causes the environmental situations to become powerful cues for the urge to smoke. Likewise, aspects of the drug-taking process, such as the manipulation of smoking materials, or the taste, smell, or feel of smoke in the throat, become associated with the pleasurable effects of smoking. Even unpleasant moods can become conditioned cues for smoking. For example, a smoker may learn that not having a cigarette provokes irritability (a common symptom of the nicotine abstinence syndrome) and smoking a cigarette provides relief. After repeated experiences of this sort, a smoker may come to regard irritability from any source, such as stress or frustration, as a cue for smoking. Although conditioning becomes an important element of drug addiction, conditioning develops only because of a pairing of the pharmacologic actions of the drug with behaviors. It has been suggested that conditioning serves to maintain nicotine use during periods of desensitization of $\alpha_{4} \beta_{2} \mathrm{nAChRs}$, in which there is a loss or decrease in the biologic response to nicotine. ${ }^{38}$ Therefore, conditioned reinforcers could be the primary motivation to smoke during periods when desensitization prevents the reinforcing effects of nicotine obtained from smoking. This relationship is renewed on a cyclic basis: After a period of abstinence, when $\alpha_{4} \beta_{2}$ nAChRs are once again sensitive, the rewarding effects of smoking are reestablished and once again paired with the sensory stimuli of tobacco smoking, and the association of these 2 factors (stimuli and reward) is again strengthened. Conditioning is a major factor that causes relapse to drug use after a period of cessation. It must be addressed as a component of counseling and behavioral therapy for drug addiction.

\section{MECHANISMS OF PHARMACOTHERAPY TO AID SMOKING CESSATION}

As detailed above, the pharmacologic effects of nicotine play a crucial role in tobacco addiction. Thus, pharmacotherapy is essential to address this component of tobacco dependence. Optimally, a pharmacologic treatment to aid smoking cessation should both block the positive reinforc- 
ing effects of nicotine and prevent or reduce the development of withdrawal symptoms. Furthermore, it would also be advantageous if the pharmacologic therapy specifically targets the receptor subtypes involved in nicotine addiction without affecting receptors that, if activated, would produce unwanted adverse effects.

The drugs currently approved by the US Food and Drug Administration (FDA) for smoking cessation include nicotine-replacement therapy (NRT) in the form of a transdermal patch, gum, nasal spray, oral inhaler, and lozenges; bupropion; and, most recently, varenicline. Nortriptyline and clonidine have been shown in clinical trials to promote smoking cessation, but they have not been approved by regulatory authorities for this purpose. ${ }^{46}$ Clinical efficacy and safety findings with these pharmacologic agents for smoking cessation are reviewed in detail elsewhere in this supplement. ${ }^{47}$

\section{NRT}

Nicotine-replacement medications may facilitate smoking cessation in several ways. The principal action is believed to be the relief of craving and withdrawal symptoms when a person stops tobacco use. ${ }^{20}$ Amelioration of withdrawal symptoms is observed with relatively low blood levels of nicotine. A second mechanism of benefit is positive reinforcement, particularly for the arousal and stress-release effects. ${ }^{20}$ The degree of positive reinforcement is related to the rapidity of absorption and the peak nicotine plasma level achieved. Thus, positive reinforcement is most relevant for short-acting, rapid-delivery formulations, such as nicotine nasal spray; however, none of the NRTs available are able to completely reproduce the rapid and high levels of plasma nicotine obtained from smoking.

A third possible mechanism of benefit has been suggested to be the potential for nicotine medications to desensitize $\alpha_{4} \beta_{2}$ nAChRs. Such desensitization would result in a reduced effect of nicotine from cigarettes, such that if a person relapses to smoking while taking NRT, the cigarette would be less satisfying and the person less likely to resume. ${ }^{20}$ Desensitization of the nicotine receptor may in itself be rewarding. As discussed previously, this may prevent the occurrence of withdrawal symptoms. Additionally, a desensitized nicotinic receptor might result in reduced receptor responsiveness to endogenously released acetylcholine, which would be expected to produce a general mood-stabilizing effect. The idea that desensitization of nicotine receptors is rewarding has not been proved and is the subject of ongoing research.

\section{Non-Nicotine Agents}

Antidepressant Agents. Bupropion is a blocker of dopamine reuptake and, to a lesser extent, norepinephrine reuptake, and it has some nicotine receptor-blocking activity ${ }^{48}$ Hence, bupropion increases brain levels of dopamine and norepinephrine, simulating the effects of nicotine on these neurotransmitters. In rats, bupropion in low doses blocks the rewarding effects of nicotine, as assessed by intracranial self-stimulation threshold, and reverses the negative affective actions of nicotine withdrawal. ${ }^{33}$ The blockade of nicotine receptors could contribute to reduced reinforcement from a cigarette in the case of a lapse. Nortriptyline, another antidepressant, is a norepinephrine reuptake blocker and, as such, would also simulate noradrenergic actions of nicotine in the brain.

Varenicline. Varenicline is an analogue of the plant alkaloid, cytisine, which has been reported to have some benefit in smoking cessation but generally poor oral bioavailability and poor brain penetration. ${ }^{49}$ Varenicline has been shown in receptor-binding studies to have a high affinity and selectivity for the $\alpha_{4} \beta_{2} \mathrm{nAChR}$ and very little effect on other nAChR subtypes ( $\alpha_{3} \beta_{4}$ and $\alpha_{7}$ homomeric receptors) or non-nicotinic receptors. ${ }^{49}$

Varenicline is a partial agonist of the $\alpha_{4} \beta_{2}$ receptor in vivo, as demonstrated by studies of dopamine release measured with microdialysis in the nucleus accumbens of conscious rats. ${ }^{49}$ Nicotine, a full agonist, causes substantial dopamine release. Varenicline, a partial agonist, produces less of a response than that of nicotine (30\% to 60\%) but at the same time blocks the effects of any nicotine added to the system. ${ }^{49}$ Thus, varenicline stimulates $\alpha_{4} \beta_{2}$ nAChRs to maintain a moderate level of dopamine release, which reduces craving and withdrawal symptoms during abstinence from smoking and also blocks the reinforcing effects of nicotine obtained from cigarette smoke in the case of relapse.

Clonidine. Clonidine is an $\alpha_{2}$-adrenergic receptor agonist that acts primarily on the brain to reduce sympathetic neural outflow. The results are sedation and anxiolysis, as well as potential hypotension, bradycardia, and dry mouth. The mechanism of clonidine's benefit in smoking cessation is thought to be related to its calming and anxiolytic effects, and this drug appears to be most useful to patients who experience a high degree of anxiety when trying to quit smoking. ${ }^{50}$

\section{ROLE OF NONPHARMACOLOGIC THERAPY TO AID SMOKING CESSATION}

Tobacco addiction, like all drug addictions, is a complex process involving the interplay of pharmacology, learned or conditioned factors, personality, and social settings. Therefore, ideal treatment for this chronic disorder involves a comprehensive approach that addresses all major aspects of tobacco addiction, both pharmacologic and nonpharmacologic. Nonpharmacologic therapy, such as individual or group counseling, is necessary to address the important role of conditioning in tobacco addiction, which is not completely addressed using pharmacologic means. Briefly, ideal characteristics of effective nonpharmacologic treatment include those that improve skills necessary to achieve and maintain abstinence, such as those that teach coping mech- 
anisms for craving and aversive abstinence symptoms. Counseling and behavioral therapy also may be tailored to address psychiatric comorbidities. The use and efficacy of various nonpharmacologic treatment approaches for smoking cessation are discussed in detail elsewhere in this supplement. $^{51}$

\section{SUMMARY AND IMPLICATIONS OF NEUROBIOLOGY FOR TREATMENT OF NICOTINE ADDICTION}

Tobacco addiction is sustained, on the whole, by the pharmacologic actions of nicotine. Addiction is mediated primarily by the actions of nicotine on brain $\alpha_{4} \beta_{2}$ nAChRs. Stimulation of these receptors results in the release of a variety of brain neurotransmitters that produce arousal, mood modulation, and pleasure. Prolonged nicotine exposure results in neuroadaptation with desensitization and an increased number of nAChRs in the brain. Smoking cessation is associated with withdrawal symptoms, which often prompt relapse. In addition to the direct role of nicotine, conditioned stimuli, such as the taste and smell of the smoke along with environmental cues, help sustain addiction to cigarette smoking. Thus, tobacco addiction is maintained not only by the positive reinforcing effects of nicotine and the conditioned stimuli associated with smoking, but also by the avoidance of the negative consequences of withdrawal.

The odds of successful smoking cessation are improved with pharmacotherapy, such as nicotine medications and bupropion. These therapies are believed to work primarily by replacing nicotine or simulating nicotinic effects in the brain, thereby reducing withdrawal symptoms experienced during cessation. Varenicline, a partial agonist at $\alpha_{4} \beta_{2}$ nAChRs, reduces withdrawal symptoms by maintaining a modest level of dopamine in the brain and, due to its receptor antagonist effects, also blocks the reinforcing effects of smoking during a lapse. This provides a novel mechanism of action that holds promise for enhancing smoking cessation success and for treating smokers who have been unable to quit using other medications. While the role of effective pharmacotherapy is essential for smoking cessation, nonpharmacologic treatment is also important to address the full spectrum of neurobiologic mechanisms that underlie tobacco addiction. Whereas much is currently known about the neurobiology of tobacco addiction, there is still much work to be done. As current areas of uncertainty become better elucidated, it is expected that treatment of nicotine addiction will be improved and abstinence will be more readily achieved on a broader scale.

\section{Acknowledgments}

The author is grateful to Marc Olmsted for editorial assistance. Additional editorial support was provided by Darlene
Benson, BSPharm, of Medesta Publications Group, and editorial support was funded in part by Pfizer Inc.

\section{AUTHOR DISCLOSURES}

Neal L. Benowitz, MD, has been a paid consultant for Acrux, Aradigm, GlaxoSmithKline, and sanofi-aventis; and is a member of the Pfizer Inc worldwide varenicline scientific advisory board.

\section{References}

1. US Department of Health and Human Services. The Health Consequences of Smoking: A Report of the Surgeon General. Atlanta, GA: US Dept of Health and Human Services, Centers for Disease Control and Prevention, National Center for Chronic Disease Prevention and Health Promotion, Office on Smoking and Health, 2004.

2. Centers for Disease Control and Prevention. Cigarette smoking among adults-United States, 2000. MMWR Morb Mortal Wkly Rep. 2002; 51:642-645.

3. Hughes JR, Gulliver SB, Fenwick JW, et al. Smoking cessation among self-quitters. Health Psychol. 1992;11:331-334.

4. Benowitz NL. Pharmacology of nicotine: addiction and therapeutics. Annu Rev Pharmacol Toxicol. 1996;36:597-613.

5. Benowitz NL. Nicotine addiction. Prim Care. 1999;26:611-631.

6. US Department of Health and Human Services. The Health Consequences of Smoking: Nicotine Addiction. Washington, DC: Office of the Surgeon General, US Dept of Health and Human Services, 1988.

7. Edwards G, Arif A, Hodgson R. Nomenclature and classification of drug- and alcohol-related problems: a shortened version of a WHO memorandum. Br J Addict. 1982;77:3-20.

8. Benowitz NL, Jacob P III. Nicotine renal excretion rate influences nicotine intake during cigarette smoking. J Pharmacol Exp Ther. 1985;234:153-155

9. Benowitz NL. Compensatory smoking of low-yield cigarettes. In: Burns DM, Shopland DR, Benowitz N, eds. Risks Associated with Smoking Cigarettes with Low Machine-Measured Yields of Tar and Nicotine. Bethesda, MD: US National Institutes of Health, National Cancer Institute, 2001:39-63.

10. Hughes JR, Hatsukami D. Signs and symptoms of tobacco withdrawal. Arch Gen Psychiatry. 1986;43:289-294.

11. Diagnostic and Statistical Manual of Mental Disorders-Fourth Edition. Washington, DC: American Psychiatric Association, 1994.

12. Heatherton TF, Kozlowski LT, Frecker RC, Fagerström KO. The Fagerström Test for Nicotine Dependence: a revision of the Fagerström Tolerance Questionnaire. Br J Addict. 1991;86:1119-1127.

13. Giovino GA, Henningfield JE, Tomar SL, Escobedo LG, Slade J. Epidemiology of tobacco use and dependence. Epidemiol Rev. 1995; 17:48-65.

14. Rigotti NA, Singer DE, Mulley AG Jr, Thibault GE. Smoking cessation following admission to a coronary care unit. J Gen Intern Med. 1991;6:305-311.

15. Walker MS, Vidrine DJ, Gritz ER, et al. Smoking relapse during the first year after treatment for early-stage non-small-cell lung cancer. Cancer Epidemiol Biomarkers Prev. 2006;15:2370-2377.

16. Himbury S, West R. Smoking habits after laryngectomy. BMJ. 1985; 291:514-515.

17. Henningfield JE, Miyasato K, Jasinski DR. Abuse liability and pharmacodynamic characteristics of intravenous and inhaled nicotine. J Pharmacol Exp Ther. 1985;234:1-12.

18. DeNoble VJ, Mele PC. Intravenous nicotine self-administration in rats: effects of mecamylamine, hexamethonium and naloxone. Psychopharmacology (Berl). 2006;184:266-272.

19. Harvey DM, Yasar S, Heishman SJ, et al. Nicotine serves as an effective reinforcer of intravenous drug-taking behavior in human cigarette smokers. Psychopharmacology (Berl). 2004;175:134-142.

20. Henningfield JE. Nicotine medications for smoking cessation. $N$ Engl J Med. 1995;333:1196-1203. 
21. Dajas-Bailador F, Wonnacott S. Nicotinic acetylcholine receptors and the regulation of neuronal signalling. Trends Pharmacol Sci. 2004;25: 317-324

22. Dani JA, De Biasi M. Cellular mechanisms of nicotine addiction. Pharmacol Biochem Behav. 2001;70:439-446.

23. Picciotto MR, Zoli M, Rimondini R, et al. Acetylcholine receptors containing the $\beta_{2}$ subunit are involved in the reinforcing properties of nicotine. Nature. 1998;391:173-177.

24. Maskos U, Molles BE, Pons S, et al. Nicotine reinforcement and cognition restored by targeted expression of nicotinic receptors. $\mathrm{Na}$ ture. 2005;436:103-107.

25. Tapper AR, McKinney SL, Nashmi R, et al. Nicotine activation of $\alpha_{4}{ }^{*}$ receptors: sufficient for reward, tolerance, and sensitization. Science. 2004;306:1029-1032.

26. Aberger K, Chitravanshi VC, Sapru HN. Cardiovascular responses to microinjections of nicotine into the caudal ventrolateral medulla of the rat. Brain Res. 2001;892:138-146.

27. Levin ED, Bettegowda C, Blosser J, Gordon J. AR-R17779, and $\alpha_{7}$ nicotinic agonist, improves learning and memory in rats. Behav Pharmacol. 1999; 10:675-680.

28. Hajos M, Hurst RS, Hoffmann WE, et al. The selective $\alpha_{7}$ nicotinic acetylcholine receptor agonist PNU-282987 [ $N$-[(3R)-1-Azabicyclo [2.2.2] oct-3-yl]-4-chlorobenzamide hydrochloride] enhances GABAergic synaptic activity in brain slices and restores auditory gating deficits in anesthetized rats. J Pharmacol Exp Ther. 2005;312:1213-1222.

29. Brody AL. Functional brain imaging of tobacco use and dependence. J Psychiatr Res. 2006;40:404-418.

30. Nestler EJ. Is there a common molecular pathway for addiction? Nat Neurosci. 2005;8:1445-1449.

31. Wonnacott S. Presynaptic nicotinic ACh receptors. Trends Neurosci. 1997;20:92-98.

32. Corrigall WA, Franklin KB, Coen KM, Clarke PB. The mesolimbic dopaminergic system is implicated in the reinforcing effects of nicotine. Psychopharmacology (Berl). 1992;107:285-289.

33. Cryan JF, Bruijnzeel AW, Skjei KL, Markou A. Bupropion enhances brain reward function and reverses the affective and somatic aspects of nicotine withdrawal in the rat. Psychopharmacology (Berl). 2003;168: 347-358.

34. Epping-Jordan MP, Watkins SS, Koob GF, Markou A. Dramatic decreases in brain reward function during nicotine withdrawal. Nature. 1998;393:76-79.

35. Wang H, Sun X. Desensitized nicotinic receptors in brain. Brain Res Brain Res Rev. 2005;48:420-437.
36. Dani JA, Heinemann S. Molecular and cellular aspects of nicotine abuse. Neuron. 1996;16:905-908.

37. Brody AL, Mandelkern MA, London ED, et al. Cigarette smoking saturates brain $\alpha_{4} \beta_{2}$ nicotinic acetylcholine receptors. Arch Gen Psychiatry. 2006;63:907-915.

38. Balfour DJ. The neurobiology of tobacco dependence: a preclinical perspective on the role of the dopamine projections to the nucleus. Nicotine Tob Res. 2004;6:899-912.

39. Donny EC, Chaudhri N, Caggiula AR, et al. Operant responding for a visual reinforcer in rats is enhanced by noncontingent nicotine: implications for nicotine self-administration and reinforcement. Psychopharmacology (Berl). 2003;169:68-76.

40. Hughes JR. Clinical significance of tobacco withdrawal. Nicotine Tob Res. 2006;8:153-156.

41. Koob GF, Le Moal M. Drug abuse: hedonic homeostatic dysregulation. Science. 1997;278:52-58.

42. Rose JE, Behm FM, Levin ED. Role of nicotine dose and sensory cues in the regulation of smoke intake. Pharmacol Biochem Behav. 1993; 44:891-900.

43. Rose JE, Behm FM, Westman EC, Johnson M. Dissociating nicotine and nonnicotine components of cigarette smoking. Pharmacol Biochem Behav. 2000;67:71-81.

44. Olausson P, Jentsch JD, Taylor JR. Repeated nicotine exposure enhances responding with conditioned reinforcement. Psychopharmacology (Berl). 2004;173:98-104.

45. Kenny PJ, Markou A. Conditioned nicotine withdrawal profoundly decreases the activity of brain reward systems. J Neurosci. 2005;25: $6208-6212$.

46. Fiore MC, Bailey WC, Cohen SJ, et al. Treating Tobacco Use and Dependence: Clinical Practice Guideline. Rockville, MD: US Dept of Health and Human Services, Public Health Service, 2000.

47. Nides M. Update on pharmacologic options for smoking cessation treatment. Am J Med. 2008;121(4A):S20-S31.

48. Slemmer JE, Martin BR, Damaj MI. Bupropion is a nicotinic antagonist. J Pharmacol Exp Ther. 2000;295:321-327.

49. Coe JW, Brooks PR, Vetelino MG, et al. Varenicline: an $\alpha_{4} \beta_{2}$ nicotinic receptor partial agonist for smoking cessation. J Med Chem. 2005;48:3474-3477.

50. Gourlay SG, Benowitz NL. Is clonidine an effective smoking cessation therapy? Drugs. 1995;50:197-207.

51. Niaura R. Nonpharmacologic therapy for smoking cessation: characteristics and efficacy of current approaches. Am J Med. 2008;121(4A): S11-S19. 